2

3

4

5

7

8

9

10

11

12

13

14

15

\title{
Impact of oral myofunctional therapy on orofacial myofunctional status and tongue strength in patients with tongue thrust
}

\author{
Mozzanica Francesco ${ }^{1,2}$, Pizzorni Nicole ${ }^{3}$, Scarponi Letizia ${ }^{3}$, Crimi Giorgia ${ }^{3}$, Schindler Antonio ${ }^{3}$
}

1. Department of Clinical Sciences and Community Health, University of Milan, Milan, Italy

2. IRCCS Multimedica, Ospedale San Giuseppe, Milan, Italy

3. Department of Biomedical and Clinical Sciences, L. Sacco Hospital, University of Milan, Milan, Italy

Impact of oral myofunctional therapy in patients with tongue thrust

\section{*Corresponding Author}

Nicole Pizzorni, SLP, PhD

Ospedale Luigi Sacco, pad 51 Phoniatric Unit, Department of Biomedical and Clinical Sciences, L.

Sacco Hospital, University of Milan, Milan, Italy

Via G.B. Grassi 74, Milan, Italy

Email: nicole.pizzorni@virgilio.it

Telephone: +390239043526

Keywords: tongue thrust; tongue strength: orofacial myofunctional therapy; malocclusion, dentition 


\section{Abstract}

Introduction: tongue thrust is a frequent clinical condition characterized by abnormal patterns of movements and altered tongue posture on the mouth floor. It might contribute to determining alterations in the maxillofacial morphology and in the development of malocclusion. Several therapeutic options are available for treatment. In particular, the orofacial myofunctional therapy (OMT) is frequently adopted even if only few studies have analyzed its efficacy using validated instruments and no information is available regarding the effect of dentition on the results obtained with OMT.

Objective: Evaluate the effect of OMT through a validated instrument and explore the role of dentition on its efficacy.

Methods: 22 consecutive patients with tongue thrust were enrolled. According to the presence of mixed or complete dentition, the cohort of patients was divided into 2 groups. Each patient underwent OMT according to the Garliner method (10 weekly sessions of 45 minutes each in hospital and daily exercises at home). The efficacy of OMT was evaluated using the Orofacial Myofunctional Evaluation with Scores (OMES), a validated protocol developed for the assessment of orofacial myofunctional disorders, and the lowa Oral Performance Instrument (IOPI) to measure the peak isometric pressure exerted by the anterior and posterior part of the tongue. Both OMES and IOPI were administered before and at the end of the treatment.

Results: A significant improvement in the OMES scores was demonstrated after OMT. No significant differences between the patients with intermediate and mixed dentition obtained both in the preand post-treatment conditions were demonstrated in the OMES scores. Similarly, a significant increase in the peak isometric tongue pressure both in the anterior and posterior part of the tongue was demonstrated after OMT in the groups. No differences between the two groups both in the preand post-treatment conditions were demonstrated in the IOPI scores.

Conclusions: OMT improves orofacial motricity and tongue strength in patients with tongue thrust regardless of the type of dentition. 
2 Patients with tongue thrust perform predominantly forward tongue movements and interpose the tongue between their teeth while speaking and swallowing, with consecutive pressure against the lingual surfaces of the anterior teeth and altered tongue position in the oral cavity. The tongue dorsum is curved downwards and the base touches the posterior part of the palate and the anterior pharyngeal wall $[1,2]$. This abnormal pattern of movement and the tongue posture on the mouth floor is considered normal in young children because it represents an oral reflex associated with suckling behavior [3], but it gradually disappears when the child develops a mature swallowing pattern. The latter is characterized by a cranial movement of the tongue and pressure on the incisive papilla $[2,4]$. The transition takes place gradually between the two phases at the age of 12-15 months along with dental eruption [1].

The persistence of tongue thrust is frequent in the pediatric population [1] and might contribute in determining alterations in the maxillofacial morphology and in the development of malocclusion because of the altered motor activity and position of the tongue in the oral cavity [2-6]. Altered tongue dynamics, in fact, is often associated with a reduced contraction of the lower jaw elevator muscles and increased activity of the perioral muscles [1]. In addition, an abnormal tongue position both at rest and during sleep reduces tongue movement and might facilitate tongue hypotonia [7]. These aspects are frequently associated with skeletal malocclusions and several studies suggested tongue thrust plays an important role in the development of anterior open bite (AOB, defined as the lack of overlap between the upper and lower incisors [2]), in the relapse of treated AOB patients [8-10], and in the development of posterior crossbite [11]. Moreover, the association of tongue thrust and AOB would also seem associated with an impairment in the oral sensory perception causing alteration in the stereognostic and two-point discrimination ability [12].

Because of its important clinical consequences, the presence of tongue thrust should be detected and treated early. Several therapeutic approaches have been reported so far [3]. In particular, the orofacial myofunctional therapy (OMT) includes different exercises whose aims are to establish a new neuromuscular pattern and to correct abnormal functional and resting postures of the tongue through improvement of its muscular 
1 proprioception, mobility and strength [2, 13, 14]. Villa et al. [7] who studied the effect of

2 OMT on tongue strength in a group of 54 children with sleep-disordered breathing

3

4

5

6

7

8

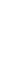
demonstrated that OMT determined an increase in tongue strength and re-established the correct tongue position. In addition, previous studies had suggested that OMT could improve tongue function during swallowing and reduce AOB [2].

OMT might be applied in combination with orthodontic treatment when tongue thrust is associated with malocclusion, or applied alone in the case of patients affected by altered tongue dynamics without malocclusion [2]. Although OMT is frequently used in everyday clinical practice, its efficacy is still a matter of debate and only few studies have analyzed its effects using validated instruments [14]. In particular, there is a scarcity of information regarding the effects of OMT on oral and facial motricity and tongue strength in patients with tongue thrust [7]. In addition, no information is available regarding the role of dentition on the effects of OMT and consequently the best timing for OMT initiation.

This prospective clinical study was performed in order to provide additional information regarding the efficacy of OMT in patients with tongue thrust. In particular, the first aim of the study was to analyze the modifications of oral and facial motricity and tongue strength following OMT using validated instruments. In addition, the correlations between oral and facial motricity and tongue strength were analyzed. Finally, the effect of dentition on the results obtained with OMT were evaluated. The importance of this study is related to better knowledge of the OMT efficacy in patients with tongue thrust being useful both in treatment planning and in pre- and posttreatment counseling. 


\section{Materials and methods}

The present study was conducted in accordance with the Declaration of Helsinki and it was previously approved by the Institutional Review Board. All the data were collected prospectively.

\section{Participants}

A total of 22 consecutive patients ( 9 males and 13 females) evaluated for tongue thrust were recruited. All the patients were referred to the Department of Phoniatrics and Speech and Language Pathology because of tongue thrust or myofunctional disorders by the Dental Department. Written informed consents were collected from patients, parents or guardians according to the age of the enrolled subjects. Inclusion criteria included abnormal tongue posture during swallowing with interposition of the tongue between the teeth and/or intermediate or complete dentition. Exclusion criteria included age less than 6 years; history of myofunctional therapy; history of swallowing, respiratory, or speech impairments; neurologic trauma, disease, or insult; head or neck surgery (with the exception of dental work); current orthodontic treatment; dental facial deficits and neurodevelopmental disorders.

The cohort of patients was divided according to the presence of intermediate or complete dentition. Intermediate dentition was defined as permanent incisors and fully erupted first molars with the presence of deciduous teeth in the buccal region. Complete dentition was defined as the presence of only permanent teeth. Ten patients (4 males and 6 females) with a mean age of $8.8 \pm 1.1$ years made up the first group (intermediate dentition). The remaining 12 patients (5 males and 7 females) with a mean age of $19.8 \pm$ 4.7 years made up the second group (complete dentition). Malocclusion was present in 10 out of 10 patients in the first group ( 9 cases of AOB and 1 case of overjet), and in 4 out of 12 patients in the second ( 4 cases of overjet).

Each patient underwent OMT according to the Garliner method [15] (10 weekly sessions of 45 minutes each in hospital and daily exercises at home). OMT consisted of isometric and isotonic exercises involving the tongue, soft palate, and lateral pharyngeal wall designed to improve suction, swallowing, chewing, breathing, and speech functions. The treatment protocol is reported in the Appendix. Patients were required to perform the 
exercises every day at home, at least three times a day, with 10-20 repetitions each

2 time. All the enrolled patients were provided with OMT by the same speech and language pathologist (SLP) with an experience of over 5 years in the treatment of orofacial myofunctional disorders. The efficacy of OMT was evaluated using a battery of examinations administered before (T0) and at the end (T1) of the treatment. The examinations were performed by an experienced SLP not involved in the treatment.

Oral Motor Clinical Assessment - Protocol of orofacial myofunctional evaluation with scores (OMES)

The assessment of oral and facial motricity before and after intervention was performed using the orofacial myofunctional evaluation with scores (OMES). This is a validated protocol developed for the assessment of orofacial myofunctional disorders (OMD). The OMES allows the examiner to express numerically on a graduated scale, his perception of oro-facial structures and functions of the patient $[16,17]$. Originally developed in Brazil and then adapted to the Italian language [18], the OMES has demonstrated good sensitivity and specificity for OMD and could be considered as a valid and useful tool in guiding treatment planning and providing outcome measures [19, 20].

The OMES protocol is divided into different sections with a maximum total score of 104 indicating the best possible condition:

- Appearance and posture: this section assesses the symmetry of the face, the position of the lips at rest, the posture of the jaw, cheeks, and tongue.

- Mobility: this section examines the ability to perform movements (protrusion, lateralization, elevation, stretching) of the lips, tongue, jaw and cheeks.

- Functions: the section assesses the orofacial functioning during breathing (nasal, oro-nasal, or oral) and swallowing (labial behavior, lingual position, presence of dysfunctional behaviors and swallowing efficiency).

- Mastication: biting, chewing pattern and the presence of dysfunctional behavior during mastication are assessed. 
2 The evaluation of the tongue strength before and after OMT was performed using the Iowa Oral Performance Instrument (IOPI). This portable device is able to measure the peak pressure (in kilopascal, $\mathrm{kPa}$ ) exerted by the tongue on an air-filled pliable plastic bulb attached to a pressure transducer. The IOPI was selected because it is one of the most commonly used measurement techniques available to objectively measure tongue strength and endurance [21].

In the present study the peak isometric tongue pressure of both the anterior and posterior part of the tongue were recorded. In order to obtain reliable measurements, the patients were seated in an upright position and were instructed to place their tongue bulb between the midline of the tongue and the hard palate just behind the upper alveolar ridge (for the measurement of the anterior peak isometric tongue pressure), and between the midline of the tongue and the area corresponding to the passage from the hard to the soft palate (for the measurement of the posterior peak isometric tongue pressure) [22]. Patients were instructed to press against the tongue bulb using as much effort as possible. At least 3 trials for both positions of the tongue bulb were collected. Patients were given a 1-min rest period between trials. The maximum isometric tongue pressure (MIP) was defined as the highest of the three peak isometric tongue pressure scores. The MIP was considered a reflection of the patient's maximum tongue blade strength in an upward direction both in the anterior and posterior part of the tongue [22].

\section{Statistical analysis}

Statistical tests were performed using the SPSS 23.0 statistical software (SPSS Inc., Chicago, IL). The sample size was calculated on the basis of a pilot study performed in our center. The post-treatment OMES score was considered the primary endpoint and a 10 point difference between the pre- and post-treatment conditions was considered clinically significant. For the study to have a power of $80 \%$, a total of 11 patients would need to be recruited to demonstrate a statistically significant difference ( $a=0.05$ ), assuming a standard deviation of 8 . The Kolmogorov-Smirnov test was used to test the normality of the distribution of OMES and IOPI scores among the patients. Since this test 
1 demonstrated that the distribution was not normal, non-parametric tests were used.

2 The Wilcoxon test was used to evaluate the differences in the OMES and IOPI scores 3 obtained before and after OMT. The Mann-Whitney test was used to evaluate the 4 presence of differences in the OMES and IOPI scores obtained in the pre- and post5 treatment conditions in patients according to the presence of complete dentition. The 6 correlation between OMES and IOPI scores was evaluated using the Spearman test. The 7 significance level was set at 0.05 for all the comparisons.

8 


\section{Results}

All the recruited patients attended the OMT regularly and none of the patients dropped out of the study or interrupted the therapy before its conclusion. None of the patients reported difficulties in performing the exercises at home.

\section{Oral Motor Clinical Assessment}

A significant improvement in the OMES total and subscale scores was demonstrated at Wilcoxon test after OMT. In particular, the median OMES total score before the OMT was 84.5, while in the post-treatment condition it was 96.5 when considering all the patients ( $p=0.001$ at Wilcoxon test). In addition, all the OMES subscale scores improved significantly after the treatment when considering the totality of the cohort (Table 1 ). Similar results were found when considering both groups, in patients with mixed or complete dentition, in fact, the OMES total and subscale scores improved significantly after OMT (Table 1). On the other hand, no significant differences in the OMES scores between the patients with intermediate and mixed dentition obtained both in the preand post-treatment conditions were demonstrated at the Mann-Whitney test $(p=0.665$ and $p=0.721$ for the pre- and post-treatment OMES total score respectively).

\section{Tongue strength}

Comparison of tongue strength between the pre- and post-treatment condition in the total cohort, as well as in both groups of patients are reported in Table 2. A significant increase in the peak isometric tongue pressure both in the anterior and posterior part of the tongue was demonstrated after OMT in both groups. When comparing the IOPI scores obtained in the patients with intermediate and complete dentition both in the pre- and post-treatment conditions, no differences were demonstrated at the MannWhitney test. In particular, no differences in the peak isometric tongue pressure of the anterior part of the tongue obtained in patients with mixed and complete dentition were found at the Mann-Whitney test $(p=0.523$ and $p=0.767$ for the pre- and post-treatment conditions respectively). Moreover, no differences in the peak isometric tongue pressure of the posterior part of the tongue between the two groups of patients were 
1 demonstrated at the Mann-Whitney test $(\mathrm{p}=0.611$ and $\mathrm{p}=0.498$ for the pre- and post-

2 treatment conditions respectively).

3

4 Correlation analysis

5 The correlation between OMES and IOPI scores obtained both in the pre- and post6 treatment conditions are reported in Table 3. No correlations were found between 7 OMES and IOPI scores obtained in the pre-treatment condition. On the other hand, 8 significant positive correlations were found between the post-treatment Mobility 9 subscale score of the OMES and the post-treatment peak isometric pressure of the 10 anterior and posterior part of the tongue. 


\section{Discussion}

2 In the present study the effect of OMT on tongue strength and orofacial motricity in patients affected by tongue thrust was studied using validated instruments (the IOPI and the OMES). The results herewith reported appear interesting and support the efficacy of OMT. First of all, none of the patients dropped out of the study or interrupted the therapy before its conclusion. In addition, none of the patients reported difficulties in performing the daily exercises at home. These data might suggest that OMT performed using the Garliner method [15], consisting of weekly sessions of 45 minutes each in hospital and daily exercises at home, is not burdensome and could be completed by patients with mixed or complete dentition.

Several protocols of OMT for tongue thrust have been described in literature [14, 23-24]. To the best of our knowledge, no study compared the efficacy of different OMT protocols. Thus, there is no evidence of the superiority of one OMT protocol over the others. The OMT protocols proposed by Garliner [15] were used for the present study. The advantage of using the Garliner method is that the OMT protocol is highly structured, making the results of the treatment reproducible regardless of the clinician who delivers it. Individualization of the therapy was, however, guaranteed by including, when needed, additional strengthening exercises to target specific orofacial deficits according to the clinical evaluation.

\section{Orofacial motricity}

The OMES total score significantly increased after OMT. No differences were found between the OMES scores obtained in patients with mixed or complete dentition, thus suggesting that this parameter did not affect oral motricity in patients with tongue thrust. The lower OMES scores obtained before OMT suggest that patients with tongue thrust demonstrated an impairment in several aspects of orofacial muscular functions, including the mastication, the appearance and posture of the face, and the mobility and the functions of lips, tongue, jaw and cheeks. This datum is not surprising since the abnormal tongue position which characterizes patients with tongue thrust is often associated with a reduced contraction of the lower jaw elevator muscles and with an increase activity of the perioral muscle [1]. In addition, altered tongue dynamics may 
1 also affect different aspects of mastication such as food transport, bolus formation, coordination with jaw movements, and pressure control against the hard palate [25]. At the end of the OMT a significant increase in OMES total and subscale scores was demonstrated. In particular, improvement in the appearance and posture subscale score suggests an improvement in the symmetry of the face and the position of the lips, jaw, tongue and cheeks at rest. The significant increase in the Mobility subscale score suggests an improvement in lips, tongue, jaw and cheeks movement, while the increase in the Functions and Mastication subscale scores suggests an improvement in the coordination between orofacial muscles, tongue, lips and masticatory muscles during deglutition. Improvement in OMES scores after OMT is consistent with previous reports $[2,16,26]$. In particular, Prado et al [26] who used the OMES in order to evaluate the effects of OMT on the masticatory function in individuals with dentofacial deformity concluded that OMT significantly improved chewing. Moreover, Van Dick et al [2] reported a positive effect of OMT on improving harmonization of orofacial functions and tongue position, strength, and posture at rest in children with AOB and a visceral swallowing pattern.

\section{Tongue strength}

The peak isometric tongue pressure significantly increased after OMT. No differences in the IOPI scores obtained in patients with mixed or complete dentition were found. These data are difficult to compare since only few studies have analyzed the peak isometric tongue pressure in patients with tongue thrust using the IOPI [27]. The low values of isometric tongue pressure found before OMT are in accordance with the results of Kurihara et al [6] who analyzed the effect of tongue thrust on tongue pressure production during swallowing in adult patients with AOB using a pressure sheet and found that patients with tongue thrust and AOD demonstrated a weaker tongue pressure than control subjects. A significant improvement in tongue strength obtained through OMT is in accordance with previous reports. Lazarus et al [28] who investigated the effects of combined IOPI and tongue depressor exercises on tongue strength, demonstrated that the combination of the exercises determined a significant improvement in IOPI scores compared to a no-exercise control group. Robbins et al [29] 
1 examined the effects of 6 weeks of IOPI exercise training on older adults (70-89 years) and reported a significant increase in tongue strength from baseline to 4 and 6 weeks of training. Clark et al [30] demonstrated a significant effect of tongue training using directional exercises on healthy adults (18-67 years), while Villa et al [7] who evaluated the effect of OMT on tongue strength in a group of children with sleep-disordered breathing reported that after 2 months' therapy with OMT the children demonstrated a significant improvement in tongue strength at the end of treatment.

It must be noted that the IOPI results obtained at the end of the treatment in patients with tongue thrust are quite similar to those reported in heathy subjects. In a recent systematic review aimed to examine the evidence for the use of the IOPI to measure strength and endurance of the tongue and hand in healthy populations and those with medical conditions, Adams et al [27] reported that the mean values of tongue strength in healthy individuals ranged from 43 to $78 \mathrm{kPa}$. It may be hypothesized that the low values of peak isometric pressure found in the anterior and posterior part of the tongue before OMT might be related to an altered tongue position, with a consequent reduction in tongue movement and consequent muscle hypotonia, caused by tongue thrust [7]. At the end of OMT the tongue strength increased reaching values considered normal for healthy individuals [27]. It may consequently be assumed that the isometric and isotonic exercises involving the tongue, soft palate, and lateral pharyngeal wall performed through the OMT could have played a role in increasing the tongue strength probably reducing tongue hypotonia related to the altered tongue position.

\section{Correlation between tongue strength and orofacial myofunctional status}

A significant positive correlation was found between the post-treatment Mobility subscale score of the OMES and the post-treatment peak isometric tongue pressure of the anterior and posterior part of the tongue. Therefore by providing a new functional scheme for tongue movement the OMT may have reduced the altered tongue position and increased its strength thus improving tongue protrusion, lateralization, elevation and stretching. This datum is in accordance with previous reports demonstrating that a higher tongue maximum pressure is related to better masticatory performance [25, 31]. 
2 The present study has several limitations. First of all, the number of enrolled subjects is small. Consequently, the results herewith reported should be considered as preliminary and a larger population is needed in order to confirm these findings. Secondly, no validated instrument, such as the Tongue Thrust Rating Scale [32], was used to assess the severity of tongue thrust. The effect of OMT on tongue strength and oral motricity might vary according to the severity of tongue thrust. However, no Italian validated version of this instrument is available so far. Consequently, no information on this point could be collected. Thirdly, the OMES and IOPI evaluations were performed by a single SLP, blind to OMT. The presence of multiple raters would have improved the reliability of these measurements. Finally, no control group was involved and no long-term results have been collected thus both these elements should contribute to using with caution the information from this study. A new study including a control group and long-term results ( 6 months and 1 year after the treatment) is required to have a better insight into the efficacy of OMT on tongue thrust.

\section{Conclusions}

The OMT is effective in improving oral motricity and tongue strength in patients with tongue thrust. The type of dentition did not affect the efficacy of OMT since no differences in the IOPI and OMES scores were demonstrated between patients with mixed or complete dentition. 


\section{Statements}

\section{Acknowledgement}

3 Nothing to declare

\section{$4 \quad$ Statement of Ethics}

5 The present study was conducted in accordance with the Declaration of Helsinki and it was

6 previously approved by the Institutional Review Board of our hospital. Written informed consents

7 were collected from patients, parents or guardians according to the age of the enrolled subjects.

\section{Disclosure Statement}

9 The authors have no conflicts of interest to declare.

10 Funding Sources

11 Nothing to declare. 


\section{Author Contributions}

2 Francesco Mozzanica: Substantial contributions to the conception of the work, analysis and 3 interpretation of data, drafting the work and revising it critically for important intellectual content; 4 final approval of the version to be published; agreement to be accountable for all aspects of the work 5 in ensuring that questions related to the accuracy or integrity of any part of the work are 6 appropriately investigated and resolved.

7 Nicole Pizzorni: Substantial contributions to the conception of the work, drafting the work and 8 revising it critically for important intellectual content; final approval of the version to be published; 9 agreement to be accountable for all aspects of the work in ensuring that questions related to the accuracy or integrity of any part of the work are appropriately investigated and resolved.

Letizia Scarponi: Substantial contributions to acquisition, analysis and interpretation of data, drafting the work and revising it critically for important intellectual content; final approval of the version to be published; agreement to be accountable for all aspects of the work in ensuring that questions related to the accuracy or integrity of any part of the work are appropriately investigated and resolved.

Crimi Giorgia: Substantial contributions to acquisition, analysis and interpretation of data, drafting the work and revising it critically for important intellectual content; final approval of the version to be published; agreement to be accountable for all aspects of the work in ensuring that questions related to the accuracy or integrity of any part of the work are appropriately investigated and resolved.

Antonio Schindler: Substantial contributions to the conception and design of the work, drafting the work and revising it critically for important intellectual content; final approval of the version to be published; agreement to be accountable for all aspects of the work in ensuring that questions related to the accuracy or integrity of any part of the work are appropriately investigated and resolved. 


\section{References (Numerical)}

1. Maspero C, Prevedello C, Giannini L, Galbiati G, Farronato G. Atypical swallowing: a review. Minerva Stomatol 2014; 63: 217-227.

2. Van Dyck C, Dekeyser A, Vantricht E, Manders E, Goeleven A, Fieuws S, et al. The effect of orofacial myofunctional treatment in children with anterior open bite and tongue dysfunction: a pilot study. Eur J Orthod 2016; 38: 227-234.

3. Inal Ö, Serel Arslan S, Demir N, Tunca Yilmaz Ö, Karaduman AA. Effect of Functional Chewing Training on tongue thrust and drooling in children with cerebral palsy: a randomised controlled trial. J Oral Rehabil 2017; 44: 843-849.

4. Peng C, Jost-Brinckmann P, Yoshida N, Miethke R, Lin C. Differential diagnosis between infantile and mature swallowing with ultrasonography. European Journal of Orthodontics 2003; 25: 451-456.

5. Machado Junior AJ, Crespo AN. Radiographic position of the hyoid bone in children with atypical deglutition. European Journal of Orthodontics 2012; 34: 83-87.

6. Kurihara K, Fukui T, Sakaue K, Hori K, Ono T, Saito I. The effect of tongue thrusting on tongue pressure production during swallowing in adult anterior open bite cases. J Oral Rehabil 2019; 46: 895-902.

7. Villa MP, Evangelisti M, Martella S, Barreto M, Del Pozzo M. Can myofunctional therapy increase tongue tone and reduce symptoms in children with sleep-disordered breathing? Sleep Breath 2017; 21: 1025-1032.

8. Ichida T, Takiguchi R, Yamada K. Relationship between the lingual-palatal contact duration associated with swallowing and maxillofacial morphology with the use of electropalatography. Am J Orthod Dentofacial Orthop 1999; 116: 146-151.

9. Fujiki T, Takano-Yamamoto T, Noguchi H, Yamashiro T, Guan G, Tanimoto K. A cineradiographic study of deglutitive tongue movement and nasopharyngeal closure in patients with anterior open bite. Angle Orthod 2000; 70: 284-289.

10. Cheng CF, Peng CL, Chiou HY, Tsai CY. Dentofacial morphology and tongue function during swallowing. Am J Orthod Dentofacial Orthop 2002; 122: 491-499.

11. Ovsenik M, Volk J, Marolt MM. A 2D ultrasound evaluation of swallowing in children with unilateral posterior crossbite. Eur J Orthod 2014; 36: 665-671.

12. Premkumar S, Avathvadi Venkatesan S, Rangachari S. Altered oral sensory perception in tongue thrusters with an anterior open bite. Eur J Orthod 2011; 33: 139-142. 
13. Smithpeter J, Covell D Jr. Relapse of anterior open bites treated with orthodontic appliances with and without orofacial myofunctional therapy. Am J Orthod Dentofacial Orthop 2010; 137: 605-614.

14. Homem MA, Vieira-Andrade RG, Falci SG, Ramos-Jorge ML, Marques LS. Effectiveness of orofacial myofunctional therapy in orthodontic patients: a systematic review. Dental Press J Orthod 2014; 19: 94-99.

15. Garliner D. Myofunctional Therapy in Dental Practice: Abnormal Swallowing Habits: Diagnosis, Treatment: A Course of Study for the Dental Practitioner and Speech Pathologis. Brooklin, NY; Bartel Dental Book Co; 1974.

16. Felício CM, Ferreira CL. Protocol of orofacial myofunctional evaluation with scores. Int J Pediatr Otorhinolaryngol. 2008; 72: 367-75.

17. De Felício CM, Medeiros APM, De Oliveira Melchior M: Validity of the "Protocol of orofacial myofunctional evaluation with scores" for young and adult subjects. J Oral Rehab 2012; 39: 744-753.

18. De Felício CM, Folha GA, Ferreira CLP, Paskay LC, Sforza C: Translation and cross-cultural adaptation of the orofacial myofunctional evaluation with scores for Italian language. CoDas 2015; 27: 575-583.

19. Scarponi L, de Felício CM, Sforza C, Pimenta Ferreira CL, Ginocchio D, et al. Reliability and Validity of the Italian Version of the Protocol of Orofacial Myofunctional Evaluation with Scores (I-OMES). Folia Phoniatr Logop 2018; 70: 8-12.

20. Machado BC, Mazzetto MO, Da Silva MA, de Felício CM. Effects of oral motor exercises and laser therapy on chronic temporomandibular disorders: a randomized study with follow-up. Lasers Med Sci 2016; 31: 945-954.

21. Adams V, Mathisen B, Baines B, Lazarus C, Callister R. A Systematic Review and Meta-analysis of Measurements of Tongue and Hand Strength and Endurance Using the lowa Oral Performance Instrument (IOPI). Dysphagia 2013; 28:350-369.

22. Youmans S, Stierwalt JAG. Measures of tongue function related to normal swallowing. Dysphagia 2006; 21: 102-11.

23. Koletsi D, Makou M, Pandis N. Effect of orthodontic management and orofacial muscle training protocols on the correction of myofunctional and myoskeletal problems in 
developing dentition. A systematic review and meta-analysis. Orthod Craniofac Res 2018; 21 : 202-215.

24. Melis M, Di Giosia M, Zawawi $\mathrm{KH}$. Oral myofunctional therapy for the treatment of temporomandibular disorders: A systematic review. Cranio 2019 17:1-7.

25. Marim GC, Machado BCZ, Trawitzki LVV, de Felício CM. Tongue strength, masticatory and swallowing dysfunction in patients with chronic temporomandibular disorder. Physiol Behav 2019; $210: 112616$.

26. Prado DGA, Berretin-Felix G, Migliorucci RR, Bueno MDRS, Rosa RR, Polizel M, et al. Effects of orofacial myofunctional therapy on masticatory function in individuals submitted to orthognathic surgery: a randomized trial. J Appl Oral Sci 2018; 26: e20170164.

27. Adams V, Mathisen B, Baines S, Lazarus C, Callister R. A systematic review and meta-analysis of measurements of tongue and hand strength and endurance using the lowa Oral Performance Instrument (IOPI). Dysphagia 2013; 28: 350-69.

28. Lazarus CL, Logemann JA, Huang C, Rademaker AR. Effects of two types of tongue strengthening exercises in young normals. Folia Phoniatr Logop 2003; 55: 199.

29. Robbins J, Gangnon RE, Theis SM, Kays SA, Hewitt AL, Hind JA. The effects of lingual exercise on swallowing in older adults. J Am Geriatr Soc 2005; 53: 1483-1489.

30. Clark HM. Specificity of training in the lingual musculature. J Speech Lang Hear Res 2012; 55: 657-667.

31. A. Yamada, M. Kanazawa, Y. Komagamine, S. Minakuchi. Association between tongue and lip functions and masticatory performance in young dentate adults. J Oral Rehabil 2015; 42: 833-839.

32. Serel Arslan S, Demir N, Karaduman AA. Reliability and validity of a tool to measure the severity of tongue thrust in children: the Tongue Thrust Rating Scale. J Oral Rehabil 2017; 44: 119-124. 


\section{Appendix. OMT therapy protocol according to Garliner method [15]}

The protocol for tongue thrust described below was the same for all patients. Patients were instructed to repeat the exercises daily at home. If necessary, the exercise introduced during a session could be repeated in the next sessions until it was adequately mastered by the patient. Additional strengthening exercises for the lips, cheeks, and tongue could be included on an individual basis, according to the results of the clinical evaluation at baseline.

Session 1. Counseling on the normal physiology of chewing and swallowing, definition of tongue thrust, etiology of tongue thrust, myofunctional disorders, results of the clinical examination, OMT therapy goals and structure, and dysfunctional oral habits to avoid. The patient is trained to identify the correct position of the tongue (alveolar papilla) on the palate at rest and during swallowing and to perform the correct tongue pattern during swallowing of saliva using 1 orthodontic elastic band on the tip of the tongue to enhance proprioception.

Session 2. The patient is instructed to maintain for 5-10 minutes the correct position of the tongue at rest using 1 orthodontic elastic band. The exercise on the correct tongue pattern during swallowing with saliva is repeated with 1 and 2 orthodontic elastic bands. Tongue strengthening exercises: repeated tongue pops, retained tongue pop hold for 10 seconds.

Session 3. The patient is instructed to maintain the correct position of the tongue at rest for 20 minutes using 1 orthodontic elastic band. The exercise on the correct tongue pattern during swallowing with saliva is repeated with 1,2 , and 3 orthodontic elastic bands. The patient is instructed to retain a sip of water at the back of the tongue for 10 seconds while keeping the tip of the tongue on the alveolar papilla. The exercise on the correct tongue pattern during swallowing is performed using water sips. Tongue strengthening exercise: retained tongue pop hold for 10 seconds. Lip strengthening exercises against resistance. Masseter strengthening exercise: effortful teeth closure.

Session 4. The patient is instructed to maintain the correct position of the tongue at rest for 30 minutes using 1 orthodontic elastic band. The exercise on the correct tongue pattern during swallowing with saliva is repeated with 1,2 , and 3 orthodontic elastic bands. The exercise on the correct tongue pattern during swallowing is performed using water sips and a cracker. Alternate chewing exercises with a cracker. Tongue strengthening exercise: retained tongue pop hold for 10 seconds. Lip strengthening exercises: lip strengthening against resistance, lip massage (cover the lower lip with the upper lip and vice versa, repeat). Masseter strengthening exercise: effortful teeth closure.

Session 5. The patient is instructed to maintain the correct position of the tongue at rest for 45 minutes using 1 orthodontic elastic band. The exercise on the correct tongue pattern during swallowing with saliva is repeated with 1,2 , and 3 orthodontic elastic bands. The exercise on the correct tongue pattern during swallowing is performed using water sips, a cracker, and midway through the meal. Tongue strengthening exercise: retained tongue pop hold for 10 seconds. Lip strengthening exercises: lip strengthening against resistance, lip massage (cover the lower lip with the upper lip and vice versa, repeat 30 times). Masseter strengthening exercise: effortful teeth closure.

Session 6. The patient is instructed to maintain the correct position of the tongue at rest for 50 minutes using 1 orthodontic elastic band and the lip closure by keeping a tongue depressor 
between the lips. The exercise on the correct tongue pattern during swallowing with saliva is repeated with 3 orthodontic elastic bands and without elastic bands. The exercise on the correct tongue pattern during swallowing is performed using water sips, a cracker, and during a whole meal. Lips strengthening exercises: lips strengthening against resistance, lips massage (cover the lower lip with the upper lip and vice versa, repeat 30 times). Masseter strengthening exercise: effortful teeth closure.

Session 7. The patient is instructed to maintain the correct position of the tongue at rest for 60 minutes using 1 orthodontic elastic band and the lip closure by keeping a tongue depressor between the lips. The exercise on the correct tongue pattern during swallowing with saliva is repeated without orthodontic elastic bands. The exercise on the correct tongue pattern during swallowing is performed using water sips, a solid food, and during a whole meal. Lip strengthening exercises: lip strengthening against resistance, lips massage (cover the lower lip with the upper lip and vice versa, repeat 30 times). Masseter strengthening exercise: effortful teeth closure.

Session 8. The patient is instructed to maintain the correct position of the tongue at rest for 60 minutes using 1 orthodontic elastic band and the lip closure by keeping a tongue depressor between the lips. The correct tongue pattern during swallowing should be checked during 2 meals. The patient and the clinician set some time periods within the day at which the patient must check and record the tongue posture at rest and during swallowing. Lip strengthening exercises: lip strengthening against resistance, lip massage (cover the lower lip with the upper lip and vice versa, repeat 30 times). Masseter strengthening exercise: effortful teeth closure.

Session 9. The patient is instructed to maintain, the correct position of the tongue at rest for 15 minutes twice a day using 1 orthodontic elastic band and the lip closure by keeping a tongue depressor between the lips. The correct tongue pattern during swallowing should be checked during all the meals. The patient must continue to check and record the tongue posture at rest and during swallowing at the defined hours. Lip strengthening exercises: lip strengthening against resistance, lip massage (cover the lower lip with the upper lip and vice versa, repeat 30 times).

Session 10. The patient is instructed to maintain, twice a day the correct position of the tongue at rest for 10 minutes using 1 orthodontic elastic band and the lips closure by keeping a tongue depressor between the lips. The correct tongue pattern during swallowing should be checked during all the meals. The patient must continue to check and record the tongue posture at rest and during swallowing at the defined hours. 